\title{
Le Thème $L$ de l'adjectif et du nom
}

\author{
Plénat, Marc \\ Axe D.U.M.A.L. \\ UMR 5263, CNRS \& Université Toulouse 2 \\ plenat@univ-tlse2.fr
}

\section{Introduction}

Le présent travail propose un premier essai d'analyse de la morphologie des substantifs déadjectivaux «savants» dans le cadre de la morphologie «thématique ». Par dérivés «savants », j'entends ici les dérivés qui, à l'ordinaire, sont formés sur un radical emprunté au latin ou imité de cette langue quand un tel radical existe (exemple : nervosité, bâti sur le même radical que lat. nervōsus, étymon de nerveux). Le manque de temps et de place me contraignent à limiter ma description aux dérivés en -ité, mais tout porte à croire que leur comportement n'est pas sensiblement différent de celui des autres déadjectivaux " savants", comme par exemple les déadjectivaux en -isme ou en -itude (cf. nervosisme, nervositude). Par morphologie «thématique », j'entends ce courant récent qui considère qu'un lexème est représenté dans le lexique par une collection de thèmes indexés (son " espace thématique ») alloués chacun à une ou plusieurs cases de son paradigme flexionnel (cf. Boyé 2000, Bonami \& Boyé 2003a, et déjà Morin 1987) et de son paradigme dérivationnel (i.e. ses dérivés immédiats, cf. Bonami, Boyé \& Kerleroux à paraitre).

Plus précisément, la présente contribution s'insère dans un ensemble de travaux sur les paradigmes flexionnel et dérivationnel de l'adjectif en français (cf. Bonami \& Boyé 2003b, 2005, Bonami, Boyé \& Tseng 2005, Boyé \& Plénat à paraître, Roché en préparation, Plénat ce volume, et déjà Morin 1993). Elle se conforme aussi à l'effort de renouvellement des données qui caractérise un certain nombre de recherches récentes (cf. Hathout \& alii 2008, Hathout \& alii à paraître). Je ne fais ici que résumer et mettre en forme une série de discussions et d'échanges que j'ai eus notamment avec G. Boyé et M. Roché (qui ne sont pas responsables de mes erreurs).

Dans la première partie, je développe rapidement l'hypothèse centrale du présent travail : les dérivés en -ité sont bâtis sur un Thème L(atin) qui, lorsqu'il est prédictible, l'est à partir du Thème 2 , c'est-à-dire du thème qui sert en particulier à former le féminin de l'adjectif. Ainsi, par exemple, sur LICENCIEUX formera-t-on ou bien licenciosité en postériorisant la dernière voyelle du Thème 2 lisãsjøz ou bien licencieusité, sur un thème identique à ce même Thème 2 .

Dans la seconde partie, je m'attarde sur les dérivés en -ité des adjectifs en -eur comme TROMPEUR et MOTEUR. D'abord pour montrer que de tels dérivés existent: MOTEUR donne motricité et TROMPEUR donne tromposité. Ensuite, parce que leur existence démontre la supériorité de l'approche adoptée sur les approches purement phonologiques: on ne voit pas quelle règle ou quelle contrainte phonologique pourrait amener les locuteurs à préférer motricité et tromposité à *motorité et *tromporité ; en revanche, le radical de ces deux dérivés se laisse analyser comme un Thème $\mathrm{L}$.

Dans la troisième partie, je montre que l'espace thématique de l'adjectif n'est pas identique à celui du substantif. Le fait, en particulier, que des substantifs comme ENTRAINEUR et PRECEPTEUR donnent des dérivés savants comme entraînorat et préceptorat (et non entraînosat et préceptriçat) montre que les Thèmes 2 de ces noms ne sont pas en -øz et -ris : ENTRAINEUSE et PRECEPTRICE sont des lexèmes à part entière et non des formes fléchies des masculins correspondants.

\section{Le Thème L}

Pour des raisons d'économie, la grammaire générative classique s'efforçait de dériver toutes les manifestations d'un même lexème à partir d'une forme sous-jacente unique. Ainsi, par exemple, à suivre 
Schane (1968), toutes les formes de l'adjectif NERVEUX peuvent être dérivées de nervoz : le timbre de la dernière voyelle est déterminé par une règle de « vowel fronting » qui l'antériorise sous l'accent et par une règle de «rounded vowel adjusment » qui la ferme devant $\mathbf{z}$, d'où le $\mathbf{o}$ de nervosité, nervosisme et le $\emptyset$ de nerveux, nerveuse ; la consonne finale, quant à elle, disparaît par application d'une règle de « truncation » devant la consonne initiale du mot suivant ou d'une règle de « final consonant deletion » en fin de groupe, d'où sa disparition dans nerveux en dehors des contextes de liaison. Cette approche a subi de multiples révisions. Ainsi, par exemple, Dell \& Selkirk (1978) ont-ils montré que la règle de «vowel fronting » devait être remplacée par une règle, «Learned Backing», conditionnée morphologiquement, qui postériorise les œ et les $\varepsilon$ des morphèmes « savants » placés devant un autre morphème « savant » (d'où le o de nervosité et nervosisme, les suffixes -eux et -ité faisant partie de la classe des morphèmes « savants »). Mais la tentation de ramener toutes les manifestations d'un lexème à une forme sous-jacente unique est restée extrêmement forte ces quarante dernières années.

L'approche adoptée ici prend le contre-pied de cette tendance. On suppose que les différents allomorphes d'un même lexème (ses «thèmes ») sont indexés dans l'entrée lexicale de celui-ci (leur ensemble forme ce que l'on appelle l' «espace thématique »du lexème) et que chacun de ces thèmes est affecté à une ou plusieurs cases de son paradigme flexionnel (sa déclinaison ou sa conjugaison) et de son paradigme dérivationnel (l'ensemble de ses dérivés directs). Dans ce cadre, les régularités phonologiques partielles sont le fait de règles de correspondance entre thèmes; les irrégularités, quant à elles, proviennent du remplissage de certaines cases de l'espace thématique ou du paradigme par des formes supplétives qui prévalent sur les formes régulières.

\subsection{L'espace thématique de l'adjectif}

Pour ce qui est de l'adjectif en français, les travaux énumérés ci-dessus se sont efforcés de démontrer que son espace thématique comportait au moins trois thèmes: le Thème 1, qui est celui qu'utilise ordinairement le masculin, le Thème 2, qui est celui qu'utilisent le féminin et la majorité des dérivés, et le Thème 3, qui est la forme que prend le radical de l'adverbe en -ment. Je propose ici d'ajouter à ces trois thèmes un thème L(atin) pour les dérivés « savants » comme les déadjectivaux en -ité. Exemples :

\section{(1) LeXeme Thème 1 Thème 2 Thème 3 Thème $L$}

\begin{tabular}{|c|c|c|c|c|}
\hline COURT & kur & kurt & kurt & kurt \\
\hline BREF & bref & brev & brijev & brev \\
\hline GRAND & grã & grãd & grãd & man \\
\hline PRUDENT & prydã & prydãt & pryda & prydãt \\
\hline IMMENSE & imãs & imãs & imãse & imãs \\
\hline SEREIN & sərẽ & səren & səren & seren \\
\hline PERENNE & peren & peren & peren & peren \\
\hline
\end{tabular}

Ces thèmes sont reliés entre eux par des règles de correspondance. Ainsi, le Thème 3 est-il par défaut identique au Thème 2 (cf. courte-ment, grande-ment, sereine-ment, pérenne-ment), mais cette règle générale se heurte à des règles particulières, comme celle qui veut que les Thèmes 2 en -ãt donnent des Thèmes 3 en a (cf. éléga-mment) ou celle qui veut que les Thèmes 2 en ãs donnent des Thèmes 3 en -ãse (cf. immensément); elle se heurte aussi à des idiosyncrasies comme le radical de briève-ment.

Ces règles de correspondance ne sont pas proprement phonologiques en ce que leur conditionnement est au moins partiellement morphologique ou lexical. Des alternances comme $\tilde{a}(\mathrm{t}) \sim$ a n'ont pas le caractère systématique que l'on attend de processus purement phonologiques: dans d'autres contextes morphologiques ãt ne perd pas son $\mathbf{t}$ devant $\mathbf{m}$ (cf. enchantement) et ã ne se dénasalise pas dans ce contexte (cf. emmener ãmne, maman mãmã, samba sãmba). La phonologie peut difficilement expliquer à 
elle seule que l'adverbe correspondant à innocent soit innocemment quand le déverbal de innocenter est innocentement, ou que précis donne précisément et préciser : précisement (cf. Boyé \& Plénat art. cit.).

Un autre trait caractéristique de ces règles de correspondance réside dans le fait qu'elles entrent en concurrence les unes avec les autres, si bien qu'il arrive fréquemment que plusieurs schèmes s'offrent au locuteur au moment où il veut créer une forme nouvelle ou une forme qu'il ignore. Beaucoup de locuteurs, par exemple, hésitent entre précocement et précocément. Les faits sont encore plus nets avec le Thème 1 et le Thème $2:$ des correspondances particulières comme if $\sim$ iv, ar $\sim \operatorname{ard}, \tilde{\varepsilon} \sim \varepsilon$ n ou $\mathrm{e} \sim \varepsilon r$ entrent en compétition avec le schème par défaut voulant que le Thème 1 et le Thème 2 soient identiques, si bien qu'au moment de donner un féminin à rosbif ou bizarre, les locuteurs hésitent entre rosbive et rosbif ou bizarre et bizarde et que, voulant trouver un masculin à pérenne lors de son débat de 2007 avec Ségolène Royal, Nicolas Sarkozy a inventé perein; d'autres disent pécunier pour pécuniaire.

\subsection{Le radical des dérivés « savants »}

Le radical des dérivés « savants » est très souvent particulier à ces dérivés, mais pas toujours. Je propose l'hypothèse que le Thème L est fondé sur le Thème 2 : sauf cas de supplétion, il est ou bien identique au Thème 2 ou bien déductible de celui-ci. Cette caractérisation permet de décrire les lexèmes recueillis dans les dictionnaires :

(2) BASE

a. ABSOLU

DOCILE

INDIGNE

MEDIOCRE

PERENNE

b. FECOND

PERVERS

(1)

NOCIF

ADULTERIN adylter $\tilde{\varepsilon}$

OPPORTUN

c.

LUBRIQUE

SEVERE

INFERIEUR

IRREEL

d. ADIPEUX

dosil

$\tilde{\varepsilon} \operatorname{din}$

peren

fekõ

perver

nosif

$$
\text { oport } \tilde{\varepsilon}
$$

sever

adipø

\section{Thème 1 Thème 2}

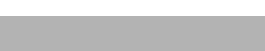

$$
\text { apsoly apsoly }
$$$$
\text { dosil }
$$

dosil

$$
\tilde{\varepsilon} \operatorname{din}
$$

medjokr medjok

peren

fekõd $\leftrightarrow$ fekõd

pervers

nosiv

$\leftrightarrow$

pervers

nosiv

(1)

NOCIVITE

$\tilde{\varepsilon}$ adylterin $\leftrightarrow$ adylterin

ADULTERINITE

oportyn

$\leftrightarrow$ oportyn

OPPORTUNITE

adaptabl adaptabl $\varnothing \sim \mathrm{i}$ adaptabil

ADAPTABILITE

lybrik lybrik k s lybris

LUBRICITE

sever

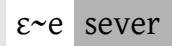

SEVERITE

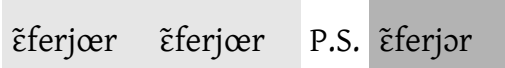

irecl ireel

P.S. ireal

adipøz

P.S. adipoz
INFERIORITE

IRREALITE

ADIPOSITE 


\begin{tabular}{|c|c|c|c|c|c|}
\hline FAMILIER & familje & familjer & P.S. & familjar & FAMILIARITE \\
\hline HUMAIN & ym $\tilde{\varepsilon}$ & ymen & P.S. & yman & HUMANITE \\
\hline e. AIGU & Egy & Egy & & aky & ACUITE \\
\hline IDOINE & idwan & idwan & & idone & IDONEITE \\
\hline NECESSAIRE & neseser & neseser & & neses & NECESSITE \\
\hline ROND & rõ & rõd & & rotõd & ROTONDITE \\
\hline VRAI & vre & vre & & ver & VERITE \\
\hline
\end{tabular}

Un certain nombre de radicaux de dérivés en -ité ne sont déductibles ni du Thème 1 ni du Thème 2 (cf. 2.e : acuité, idonéité, etc.), ce sont des cas de supplétion. En dehors de ces cas, assurément minoritaires, le Thème L est identique au Thème 2 (cf. 2.a absoluité, docilité, etc., et 2.b fécondité, perversité, etc.) ou en diffère réglément, par insertion d'un /i/ à l'intérieur d'un groupe /bl/ final (cf. 2.c adaptabilité), par assibilation d'un /k/ final (cf. 2.c lubricité), par «ajustement de /e/» (cf. 2.c sévérité) ou par «postériorisation savante» de la voyelle moyenne de la dernière syllabe (cf. 2.c infériorité, irréalité, 2.d adiposité, familiarité, humanité). Le fait que c'est bien le Thème 2 qui sert de base n'est décelable que lorsque l'adjectif n'est pas épicène et que le féminin diffère du masculin par la présence d'une consonne finale (cf. 2.b féconde, perverse, 2.d adipeuse), avec, parfois, une différence de timbre vocalique (cf. 2.b adultérine, opportune, 2.d humaine, familière), ou encore par une consonne finale différente de celle du masculin (cf. 2.b : nocive).

Les correspondances réglées entre le Thème 2 et le Thème $\mathrm{L}$ ont pour origine des processus phonologiques, mais leur conditionnement actuel n'est plus proprement phonologique. Le locuteur détermine une liste de lexèmes et de suffixes passibles des diverses règles de correspondance. Ces listes évoluent dans le temps et varient de locuteur à locuteur. Les alternances ne s'appliquent pas aveuglément : à humble correspond normalement humilité et non humbilité, antique donne antiquité et non anticité, amène fait aménité, pas amanité, et perennité, dans certains dialectes au moins, se prononce avec un $\grave{e}$ ouvert et non un é fermé devant le $\mathbf{n}$.

Les trois schèmes radical supplétif + ité, Thème 2 modifié + ité et Thème $2+$ ité sont en concurrence les uns avec les autres quand il s'agit de créer des formes nouvelles :

1 Les radicaux supplétifs tendent à être remplacés par des Thèmes 2. Cette évolution peut affecter des dérivés d'adjectifs dont le sens s'est spécialisé : appliqué à un angle, AIGU donne aiguïté au lieu de acuité ; quand il décrit un phonème, SOURD donne sourdité au lieu de surdité. Il arrive surtout que des locuteurs ignorent la forme supplétive héritée et lui substituent le Thème 2 de l'adjectif. Il conviendrait de mener une enquête pour connaître l'ampleur de ce phénomène dans le lexique non normé, mais son existence ne fait aucun doute : il n'est pas difficile de trouver sur la Toile des exemples comme idoinité au lieu de idonéite, comme rondité au lieu de rotondité, ou comme vidité au lieu de vacuité ${ }^{1}$. Il arrive aussi, plus rarement, que le radical d'origine latine soit concurrencé par un radical d'une autre origine, grecque par exemple. Ainsi nigrité est-il concurrencé non seulement par noirité, mais aussi et surtout par mélanité.

2 Dans quelques cas, il arrive qu'un radical supplétif soit concurrencé par un Thème 2 modifié régulièrement: on trouve par exemple un nombre appréciable d'occurrences de aimabilité (qui est dans Bossuet) en lieu et place de amabilité et un tout petit nombre d'attestations de humbilité pour humilité.

3 De la même façon, les modifications qui altèrent régulièrement le Thème 2 peuvent être absentes. Cela est particulièrement vrai pour les formes normalement soumises à la postériorisation savante. 
Si l'on en croit par exemple Google, près d'un tiers des locuteurs préfèrent moelleusité à moellosité et près d'un dixième réfractairité à réfractarité. Roché (à paraître) signale que Nyrop notait déjà à propos de l'alternance -aire -ar- que «[s]ous l'influence du mot primitif, ai pénètre parfois dans le dérivé » et qu' ' [o]n trouve ainsi autoritairisme, unitairisme, utilitairisme, volontairiat, à côté de autoritarisme, unitarisme, utilitarisme, volontariat ».

4 Mais, à l'inverse, une modification régulière peut affecter un lexème qui historiquement ne devrait pas la subir : quand il est affublé du suffixe «savant»-ité au lieu du suffixe "populaire »-eté, débonnaire fait plus souvent débonnarité que débonnairité comme s'il comprenait le suffixe -aire, alors qu'étymologiquement, -aire y est non pas un suffixe, mais un nom (area ou ager, cf. TLF s.v. aire). On trouve aussi quelques attestations de témérarité au lieu de témérité et de nécessarité pour nécessité.

5 Dans certains cas, les trois schèmes sont attestés. Par exemple, DOULOUREuX donne à la fois dolorosité, doulourosité et douloureusité, et RIGOUREUX : rigorosité, rigourosité et rigoureusité. Des formes comme rigourosité et doulourosité sont particulièrement intéressantes en ce qu'elles montrent que la postériorisation savante n'est pas une pure et simple reprise du lexème latin : dans ces formes, seule la finale du radical est latinisée.

Ainsi les dérivés en -ité se laissent-ils décrire assez commodément dans le cadre d'une morphologie thématique reconnaissant l'existence d'un thème L particulier aux déadjectivaux savants. Je me suis efforcé de montrer que, hormis les cas de supplétion, ce Thème L est fondé sur le Thème 2, qu'il reprend ou modifie réglément. Mais cette démonstration repose sur un certain nombre de présupposés sur ce qu'est la phonologie (par exemple l'idée que le conditionnement des processus phonologiques est purement phonologique ou l'idée que les représentations phonologiques sont relativement « concrètes »). Dans d'autres conceptions de la phonologie, il serait sans doute tout à fait possible de soutenir que le radical des dérivés en -ité examinés ci-dessus est issu d'une forme sous-jacente commune aux formes fléchies et aux formes dérivées. Mais, comme on va le voir, les dérivés en -ité bâtis sur des adjectifs en -eur ou en -teur rendent cette conception de la représentation lexicale des lexèmes assez difficile à soutenir.

\section{Les dérivés en -ité des adjectifs en -EUR}

Les dictionnaires donnent très peu d'indications sur les noms en -ité issus de bases adjectivales en -EUR. Sauf erreur de ma part, les seuls dérivés de ce type dans le TLF sont motricité (de MOTEUR) et deux lexèmes apparentés : psychomotricité et vasomotricité (de PSYCHOMOTEUR et VASOMOTEUR). Le seul autre dérivé en -ité sur base en -EUR est autorité, mais AUTEUR n'est pas un adjectif et ne fait que rarement autrice au féminin ; autorité, qui, en outre, est emprunté au latin, n'a donc pas à entrer en ligne de compte ici. Le seul indice fourni par les dictionnaires suggère donc bien que les dérivés en -ité des adjectifs en -teur sont formés sur le Thème 2 de l'adjectif (motris est le Thème 2 de l'adjectif MOTEUR), mais cet indice est isolé et ne concerne que les dérivés en -EUR sur thème de supin: on ne dispose dans les dictionnaires d'aucune indication sur les adjectifs en -EUR formés sur un thème de présent. Force était donc de mener une enquête

\subsection{Les dérivés d'adjectifs en -EUR sur thème de supin}

Nous avons recherché sur Google en français les éventuels dérivés en -ité d'adjectifs en -TEUR en partant de la liste de ces adjectifs dans le $T L F^{2}$. Nous avons trouvé une vingtaine de formes :

(3) Base

BIMOTEUR -tricité -torité -teurité Base

0 NOVATEUR -tricité -torité -teurité

$4 \quad 0 \quad 0$ 


\begin{tabular}{|c|c|c|c|c|c|c|}
\hline CASTRATEUR & 1 & 0 & 0 & PROCREATEUR & 1 & 0 \\
\hline CONDUCTEUR & 12 & 0 & 0 & PROLIFERATEUR & 1 & 0 \\
\hline CREATEUR & 1 & 0 & 0 & PSYCHOMOTEUR & 375.000 & 0 \\
\hline DIRECTEUR & 1 & 0 & 0 & QUADRIMOTEUR & 1 & 0 \\
\hline ELECTROMOTEUR & 4 & 0 & 0 & RECEPTEUR & 1 & 0 \\
\hline GENERATEUR & 2 & 0 & 0 & REDEMPTEUR & 1 & 0 \\
\hline INNOVATEUR & 1 & 0 & 0 & SALVATEUR & 1 & 0 \\
\hline LOCOMOTEUR & 72 & 0 & 0 & SENSORIMOTEUR & 444 & 0 \\
\hline MODERATEUR & $2(?)$ & 0 & 0 & ULTRAPROTECTEUR & 1 & 0 \\
\hline MOTEUR & 935.000 & $4(?)$ & 0 & VASOMOTEUR & 7010 & 0 \\
\hline NEGATEUR & 71 & 0 & 0 & & & \\
\hline
\end{tabular}

Abstraction faite de quelques exemples de motorité ${ }^{3}$, qui ne pèsent pas bien lourd en face des milliers de motricité, tous les dérivés que nous avons trouvés sont en -tricité et non en -torité ou en-teurité.

Un tiers d'entre eux sont apparentés à motricité. Il s'agit essentiellement de psychomotricité et de vasomotricité, déjà cités, et de quelques dérivés bâtis sur le même modèle : électromotricité, locomotricité, sensorimotricité. Ce sont là les dérivés les mieux attestés ${ }^{4}$.

Les données rassemblées nous apprennent aussi que des langages de spécialité autres que la biologie et la psychologie peuvent recourir sans effet particulier à des dérivés en -tricité pour nommer des concepts nouveaux. La physique fournit conductricité, la biologie réceptricité, les mathématiques génératricité, et la philosophie négatricité. L'un (au moins) de ces lexèmes est relativement ancien : on rencontre conductricité dans un traité de sidérotechnie de 1812. Un autre (au moins) apparaît sous une plume fameuse : on a trouvé génératricité dans une citation d'Edgar Morin.

D'autres exemples sont de simples essais sans lendemain (innovatricité, novatricité), des occasionnalismes appelés par la présence de l'adjectif correspondant dans le contexte (ultraprotectricité), des parodies du style savant (prolifératricité) ou emphatique (rédemptricité). Ce sont des mots qui ne sont probablement pas destinés à perdurer (mais qui peuvent resurgir à tout moment).

Le problème, cependant, n'est pas là. Il s'agissait seulement de savoir si, étant donné un adjectif en -TEUR, le dérivé correspondant était en -torité, en -teurité ou en -tricité. Qu'ils soient ancrés dans le lexique, candidats à une entrée, ou simple fantaisie, tous les dérivés recueillis ou presque ${ }^{5}$ plaident sans équivoque en faveur de la dernière possibilité.

\subsection{Les dérivés d'adjectifs en -EUR sur thème de présent}

Nous avons également recherché sur Google en français les éventuels dérivés en -ité d'adjectifs en -EUR construits sur des thèmes de présent en partant de la liste de ces adjectifs dans le $T L F^{6}$. Nous avons trouvé là aussi une vingtaine de formes : 
(4) Base

\begin{tabular}{|c|c|c|c|c|c|c|c|c|}
\hline BANDEUR & 5 & 0 & 0 & 0 & MAGOUILLEUR & 1 & 0 & 0 \\
\hline BLAGUEUR & 2 & 0 & 0 & 0 & PARLEUR & 1 & 0 & 0 \\
\hline BRANLEUR & 3 & 0 & 0 & 1 & PETIT JOUEUR & 0 & 1 & 0 \\
\hline CHARMEUR & 3 & 0 & 0 & 0 & PLEUREUR & 2 & 0 & 0 \\
\hline CHIEUR & 5 & 54 & 0 & 2 & RALEUR & 2 & 0 & 0 \\
\hline CRANEUR & 1 & 3 & 0 & 0 & REVEUR & 2 & 0 & 0 \\
\hline EMMERDEUR & 1 & 1 & 0 & 0 & SONGEUR & 1 & 0 & 0 \\
\hline GAFFEUR & 1 & 1 & 0 & 0 & TAPAGEUR & 3 & 0 & 0 \\
\hline FRIMEUR & 2 & 1 & 0 & 0 & TROMPEUR & 1 & 0 & 0 \\
\hline GLANDEUR & 3 & 0 & 0 & 0 & & & & \\
\hline
\end{tabular}

Abstraction faite de deux occurrences de chieurité (qui est peut-être en fait dérivé du nom CHIEUR), tous les dérivés déadjectivaux que nous avons trouvés sont bâtis ou bien sur un thème identique au Thème 2 de l'adjectif (c'est le cas notamment de la majorité des dérivés de CHIEUR, qui donne le plus souvent chieusité), ou bien sur un Thème L spécifique dérivé de ce même Thème 2 par postériorisation savante (pratiquement tous les adjectifs de la liste donnent au moins un dérivé en -osité). Nous n'excluons pas que tel ou tel de ces dérivés soit construit sur le nom en -EUSE plutôt que sur l'adjectif, mais les données sont parfaitement claires : les radicaux sur lesquels sont bâtis ces dérivés en -ité ne sont pas dérivables phonologiquement du Thème 1 de l'adjectif.

Aucun de ces dérivés n'a sans doute vocation à s'incruster dans le lexique, mais leur légitimité comme occasionnalismes ne fait pour moi aucun doute. Je ne résiste pas à la tentation de citer l'un d'entre eux dans son contexte parodique :

On peut penser que les deux facteurs sont importants : aussi bien l'acte que la pensée.

On peut aussi imaginer que ces deux facteurs sont liés par une sorte de formule

isTromper $=\left(\right.$ penseeTrompeuse ${ }^{*}$ poidsPensees + acteTrompeur*poidsActes $)>1$

avec poidsPensees et poidsActes dans [0,1] (intervalle permettant de donner un rapport de poids entre les deux facteurs) ainsi que penseeTrompeuse et acteTrompeur dans un intervale $[0,1]$ permettant d'exprimer la "tromposité" de la pensée/l'acte en question.

www.sorties.org/forum_messages.php?topic $=1849$

À partir du moment où le caractère trompeur d'une pensée ou d'un acte peut être mesuré, la tromposité devient un concept qui mérite une dénomination. La trompologie n'a pas la légitimité de la sidérotechnie ; mais cette illégitimité ne s'étend pas aux dénominations des pseudo-concepts qu'elle utilise : du point de vue de la morphologie, tromposité est aussi bon que conductricité. 


\subsection{Le Thème $L$ des adjectifs en -EUR et -TEUR.}

Du point de vue de la morphologie « thématique », les faits qui viennent d'être décrits sont attendus : les dérivés déadjectivaux en -ité sont bâtis sur un Thème L lui-même issu du Thème 2 soit directement, soit par l'intermédiaire de la règle de "postériorisation savante ». Bâti sur un thème identique au Thème 2 de CONDUCTEUR (kõdyktris), conductricité est entièrement parallèle à adultérinité ; tromposité, de son côté, est construit sur un thème qui ne s'écarte du Thème 2 trõpøz que par la postériorisation de sa dernière voyelle, et ressemble ainsi à s'y méprendre à adiposité ; enfin, le fait que l'on observe parfois des formes du type ${ }^{\circ}$ trompeusité (cf . chieusité en (4)) s'explique par les intermittences de la règle de postériorisation savante, qui rend compte aussi des formes (réputées fautives) du type ${ }^{\circ}$ adipeusité :

(5) BASE

$\begin{array}{llllll}\text { ADULTERIN } & \text { adylter } \tilde{\varepsilon} & \text { adylterin } & \leftrightarrow & \text { adylterin } & \text { ADULTERINITE } \\ \text { ADIPEUX } & \text { adipø } & \text { adipøz } & \text { P.S. } & \text { adipoz } & \text { ADIPOSITE } \\ \text { ADIPEUX } & \text { adipø } & \text { adipøz } & \leftrightarrow & \text { adipøz } & { }^{\circ} \text { ADIPEUSITE } \\ \text { CONDUCTEUR } & \text { kõdyktœr } & \text { kõdyktris } & \leftrightarrow & \text { kõdyktris } & \text { CONDUCTRICITE } \\ \text { TROMPEUR } & \text { trõpœr } & \text { trõpøz } & \text { P.S. } & \text { trõpoz } & \text { TROMPOSITE } \\ \text { TROMPEUR } & \text { trõpœr } & \text { trõpøz } & \leftrightarrow & \text { trõpøz } & { }^{\circ} \text { TROMPEUSITE }\end{array}$

En revanche, dans un cadre qui ne renonce à l'hypothèse de la forme sous-jacente unique que dans les cas extrêmes, la solution serait certainement plus compliquée. Dans un tel cadre, en effet, des dérivés comme adultérinité et adiposité seraient construits sur des radicaux (adylterin, adipoz) qui seraient tout bonnement la forme-sous-jacente unique de l'adjectif ou qui en dériveraient. Les dérivés de ce type ne pourraient pas servir de modèles pour conductricité et tromposité, pour lesquels on serait bien en peine de proposer des formes sous-jacentes qui donnent en même temps conducteur et trompeur ${ }^{7}$. Dans la mesure où les curiosités comme conductricité et tromposité sont, semble-t-il, peu anciennes ou récentes et peu diffusées ou même rarissimes, on ne voit pas comment les locuteurs pourraient déterminer qu'elles doivent être construites sur les radicaux kõdyktris et trõpoz (ou trõpøz) plutôt que sur les radicaux par défaut kõdyktœr et trõpœr ou sur les radicaux modifiés kõdyktor et trõpor. Pour que le cas général (adultérinité et adiposité) puisse servir de modèle au cas particulier (conductricité et tromposité), il faut que des radicaux comme adylterin ou adipoz soient identifiés comme représentant un thème particulier fondé sur celui qu'utilise le féminin et non comme une forme sous-jacente indifférenciée accommodée par la phonologie.

\section{Les paradigmes dérivationnels du nom et de l'adjectif}

Obstacle apparent à l'hypothèse avancée ci-dessus, certains Thèmes L de dérivés en -TEUR ou -EUR sont formés non pas sur des thèmes en -euse et en -trice, mais sur des thèmes en -eur et en -teur (cf. gouvernorat et électorat). Est-ce à dire que le Thème L est parfois relié au Thème 1 ? Je ne le crois pas. Les dérivés en -orat et -torat sont construits non pas sur des adjectifs, mais sur des substantifs. Dans le substantif, les oppositions -eur / -euse et -teur / -trice ont une utilisation non pas flexionnelle mais dérivationnelle. Le Thème 2, dans ce cas, est identique au Thème 1, et il est donc normal que gouverneur donne gouvernorat et électeur, électorat. Le français n'est pas encore "politiquement correct » au point d'avoir forgé gouvernosat 'dignité de gouverneuse' ou électriçat 'ensemble des électrices', mais les adverbes en -ment montrent bien que les noms masculins et féminins constituent des lexèmes distincts et non des flexions d'un même lexème. 


\subsection{Les dérivés en -orat}

On sait que les noms en -EUR bâtis sur un thème de supin donnent très facilement des dérivés en -orat qui dénotent la dignité ou la fonction exercée par le référent de la base, la durée du mandat ou le territoire sur lequel il s'exerce, le siège où résident les dignitaires ou l'ensemble de ceux-ci (cf. électorat, monitorat, préceptorat, professorat, provisorat, tutorat etc.). Ces formes sont concurrencées par des formes en -oriat, -eurat et même -euriat, ce qui ne surprend guère vu les intermittences de la règle de «postériorisation savante ». Voici un échantillon de notre récolte :
(6) AMATEUR
amatorat
amateurat amateuriat
ASSESSEUR assessorat assessoriat assesseurat
AUDITEUR auditorat auditoriat
COMMENTATEUR commentatorat
commentateuriat
CONDUCTEUR conductorat
DICTATEUR dictarorat dictatoriat dictateuriat
(7) ENTRAINEUR entraînorat
ENTREPRENEUR entreprenorat entreprenoriat entrepreneurat entrepreneuriat
MENEUR menorat
VISITEUR visitorat visitoriat

On sait peut-être moins que certains dérivés en -EUR construits sur des thèmes de présent donnent eux aussi des dérivés en -orat. Dell et Selkirk (1978 : note 34) ne citent gourvernorat. Mais on trouve aussi :

Il arrive même que des noms en -œr qui renvoient aux titulaires d'une fonction sans être pour autant analysables comme des dérivés de thèmes de présent donnent des dérivés en -orat ${ }^{8}$ :

(8) INGENIEUR ingéniorat ingénioriat ingénieurat ingénieuriat

LEADER leadorat

SUPPORTER supportorat supportoriat supporteuriat

WEBMASTER webmastorat webmastoriat

Nous ne nous intéresserons pas ici aux variations de la terminaison. Ce qui nous intéresse, c'est que les dérivés listés sont du type -orat et -torat, et non du type -osat et -triçat. Ce qui peut paraître surprenant, dans la mesure où certains au moins de ces noms ont des féminins en -euse (entraîneuse, entrepreneuse, meneuse, visiteuse et même parfois leadeuse) ou en -trice (auditrice, commentatrice, conductrice, supportrice et même parfois webmestrice).

La solution de ce semblant d'énigme réside très probablement dans la catégorie syntactico-sémantique des bases (Roché en préparation). Les bases comme celles de (6), (7) et (8) qui donnent des dérivés en -orat sont des "classifiants » (des noms de gens de métier, de fonctionnaires, de dignitaires), alors que les bases réunies en (3) et (4) qui donnent des dérivés en -osité et -tricité ont une valeur «qualifiante » ou « caractérisante » (substantifs ou adjectifs, elles « désignent ou qualifient une personne d'après un trait 
de caractère ou de comportement » (Roché, ibid.). On a tout lieu de penser que le féminin d'un nom classifiant ne fait pas partie du paradigme flexionnel de ce nom, mais de son paradigme dérivationnel: c'est un nom dénotant soit la femelle d'un agent soit un agent féminin qui n'a pas les mêmes capacités référentielles que le masculin correspondant, lequel désigne un agent mâle ou de sexe indifférent. En revanche, dans les adjectifs, le féminin fait partie de la flexion du lexème.

À partir de là, la solution qui s'impose consiste à supposer que le Thème 2 des substantifs masculins en -EUR et en -TEUR est identique au Thème 1. (En règle générale, la forme libre d'un nom se terminant par une consonne sert aussi de radical quand le nom est suffixé). Quand la règle de "postériorisation savante» s'applique, le Thème L de ces noms est en -or ou en -tor. En revanche, le Thème 2 des «qualifiants » en -EUR et en -TEUR (fondamentalement des adjectifs) est, comme nous l'avons dit, en -oz et en -tris, d'où les dérivés en -osité et en -tricité :

\begin{tabular}{|c|c|c|c|c|c|}
\hline BASE & Thème 1 & Thème 2 & & Thème L & DERIVE \\
\hline CONDUCTEUR $_{\mathrm{A}}$ & kõdyktœr & kõdyktris & $\leftrightarrow$ & kõdyktris & CONDUCTRICITE \\
\hline TROMPEUR $_{\mathrm{A}}$ & trõpœr & trõpøz & P.S. & trõpoz & TROMPOSITE \\
\hline CONDUCTEUR $_{\mathrm{Nm}}$ & kõdyktœr & kõdyktœr & P.S. & kõdyktor & CONDUCTORAT \\
\hline ENTRAINE & ãtrenœr & ãtrenœr & P.S. & ãtrenor & ENTRAINORAT \\
\hline
\end{tabular}

Cette hypothèse comporte une conséquence que nous ne sommes pas en mesure de vérifier directement. Si les noms classifiants féminins sont des lexèmes à part entière, on s'attendrait à ce qu'ils puissent servir de base à la dérivation en -at. La fonction d'entraîneuse devrait être l'entrainosat, l'ensemble des conductrices devrait être le conductricat ou le conductriçat. Je n'ai pas été capable jusqu'à présent de trouver de bons exemples de ce type. Il faut avouer que les chances d'en trouver un jour sont plutôt minces. Les noms masculins de dignitaires ou de termes apparentés s'appliquent en général à l'ensemble des titulaires, quel que soit leur sexe, quand ils servent de base aux dérivés en -at; le conductorat ou l'entraînorat englobent les conductrices et les entraîneuses (au sens sportif du terme). Il faudra que les féministes soient bien puissant(e)s pour que le féminin devienne le terme non marqué de l'opposition et que l'on dise électriçat pour électorat. En outre, les fonctions réputées spécifiquement féminines sont peu nombreuses et leur indignité supposée s'accorde mal avec le suffixe -at (il est difficile d'élever quelqu'un au blanchissosat ou au tapinosat ${ }^{9}$ ). C'est pourquoi nous avons cherché une preuve indirecte, dans une autre dérivation.

\subsection{Le cas des adverbes en -ment}

L'étude des adverbes en -ment (Boyé \& Plénat à paraitre) conduit à la même conclusion que celle des substantifs en -ité et en -at. Ces adverbes sont à l'ordinaire construits sur des adjectifs, mais ils le sont à l'occasion sur des substantifs, notamment dans les formules de politesse du type Xment vôtre en fin de lettre avant la signature, et dans les expressions du type Xment parlant. Que la base soit adjectivale ou substantivale, l'allomorphe sélectionné est, dans le cas général, identique au Thème 2 : COUSIN donne cousinement (dans cousinement vôtre, Jules, par exemple), comme MESQUIN donne mesquinement. Dans le cas des noms d'êtres sexués qui admettent un nom de femelle à côté du nom de mâle, l'adverbe tiré du second est généralement identique à celui qui est tiré du premier (COUSINE donne cousinement dans cousinement vôtre, Julie). Si, cependant, ce qui vient d'être dit est exact, on s'attend à ce que les lexèmes en -TEUR et en -EUR donnent des adverbes différents en fonction de leur catégorie syntaxique (substantif ou adjectif) et, pour les substantifs, en fonction de leur genre (masculin ou féminin). Les prédictions sont les suivantes: 


$$
\text { Base Thème 2 Adverbe }
$$

$\begin{array}{llll}\text { MOTEUR }_{\mathrm{A}} & \text { motris } & \text { MOTRICEMENT } & \text { motrismã } \\ \text { MODERATEUR }_{\mathrm{Nm}} & \text { moderatœr } & \text { MODERATEUREMENT } & \text { moderatœrmã } \\ & & & \\ \text { MODERATRICE }_{\mathrm{Nf}} & \text { moderatris } & \text { MODERATRICEMENT } & \text { moderatrismã }\end{array}$

$\begin{array}{llll}\text { TROMPEUR }_{\mathrm{A}} & \text { trõpøz } & \text { TROMPEUSEMENT } & \text { trõpøzmã } \\ \text { ENTRAINEUR }_{\mathrm{Nm}} & \text { ãtrenœr } & \text { ENTRAINEUREMENT } & \text { ãtrenœrmã } \\ & & & \\ \text { ENTRAINEUSE }_{\mathrm{Nf}} & \text { ãtrenøz } & \text { ENTRAINEUSEMENT } & \text { ãtrenøzmã }\end{array}$

Les adjectifs ayant pour Thème 2 des formes en -tris et en $-\varnothing z$, on s'attend à ce que MOTEUR donne motricement et TROMPEUR, trompeusement. Dans le cas des substantifs, en revanche, le Thème 2 n'étant pas différent du Thème 1, on s'attend à ce que MODERATEUR et ENTRAINEUR fassent modérateurement et entraîneurement, tandis que MODERATRICE et ENTRAINEUSE devraient faire modératricement et entraîneusement.

À notre connaissance, ces prédictions correspondent à la pratique des locuteurs. Pour ce qui est des déadjectivaux, il est inutile de donner des exemples du type de trompeusement. Trompeusement lui-même figure dans le $T L F$, comme rageusement, rêveusement, songeusement et plusieurs autres. Motricement ne figure pas dans le $T L F$, mais on le trouve sur la Toile :

La souris, qui a exploré perceptivement et motricement son environnement, [...]

[...] avec des réponse "motricement violentes", inadaptées, [...]

Mais "psycho-motricement" pensez vous qu'il est possible pour un corps lambda d'assimiler plusieurs langages martiaux simultanément?

(Moteurement apparaît aussi, mais cet adverbe est construit sur le substantif : on dira par exemple que tel dispositif mécanique est « excellent esthétiquement et moteurement »).

Pour ce qui est des dénominaux, on trouve de bons exemples de contraste entre adverbes construits sur un nom en -TEUR masculin et adverbes construits sur le nom correspondant en -TRICE. Boyé \& Plénat (à paraître) donne des exemples de animateurement et animatricement. On constate aussi que les modérateurs de listes de discussion terminent parfois leurs interventions par modératricement vôtre quand ce sont des modératrices ( "(Une dernière fois) modératricement, Fannie ", "Modératricement vôtre mais pas trop modérée »), et par modérateurement vôtre quand ce sont des modérateurs («Leptijo, modérateurement vôtre », " Modérateurement tien»). Je n'ai pas, jusqu'à présent, déniché de paires analogues pour les noms en -eur, mais on trouve par exemple entraîneurement à côté de danseusement :

il y a des clubs en "recherche" genre troye (mais ps de grosse ecurie)

je leur propose govou et j'ai une reponse assez rapide "ils ne sont pas intereses"

bug ou pas ?????????

ENTRAINEUREMENT VOTRE !!!!!!!! [signé C., supporter]

Cela serait sympa que tu nous tiennes au courant de ta grande Aventure.

Danseusement Vôtre [signé Muscdorient]

Ainsi y a-t-il bien lieu de croire qu'utilisées flexionnellement par l'adjectif, les oppositions -eur / -euse et -teur / -trice le sont dérivationnellement par le nom. Les espaces thématiques et les paradigmes constructionnels du nom et de l'adjectif ont très probablement la même structure, mais leurs cases ne sont pas remplies identiquement. Le Thème 2 de l'adjectif diffère systématiquement du Thème 1 et cette 
différence se répercute sur le Thème L (moteur, motrice, motricement, motricité ; trompeur, trompeuse, trompeusement, tromposité). Le Thème $2 \mathrm{du}$ nom est en revanche systématiquement identique au Thème 1, mais ces deux thèmes sont différents suivant que l'on a affaire à un masculin ou à un féminin. D'où des séries qui diffèrent en fonction du genre de la base (modérateur, modérateurement, modératorat, entraîneur, entraîneurement, entraînorat vs. modératrice, modératricement, entraîneuse, entraîneusement). Le parallélisme de ces séries fait penser que modératriçat (ou modératricat) et entraînosat sont légitimes du point de vue du système.

\section{Conclusion}

Nous avons considéré non pas les radicaux adjectivaux «savants » en tant que tels, mais l'ensemble des radicaux adjectivaux apparaissant devant le suffixe «savant»-ité. Cet ensemble est constitué de trois sous-ensembles correspondant chacun à un schème productif. On y trouve des emprunts (e.g. rigoros-), des latinisations partielles du thème qui sert à former le féminin de l'adjectif (e.g. rigouros-) et des radicaux identiques à ce dernier (e.g. rigoureus-). Cette caractérisation de la classe des radicaux apparaissant devant -ité repose sur les ressemblances que ceux-ci entretiennent avec le thème de féminin : hormis les cas de supplétion (emprunts), ces radicaux ou bien sont identiques aux thèmes de féminin ou bien n'en diffèrent que par des modifications réglées (épenthèse de $i$, assibilation, postériorisation des voyelles moyennes, ajustement de é).

Cette caractérisation permet de prédire le comportement de deux classes d'adjectifs dont les dérivés sont absents des dictionnaires et du lexique intériorisé du plus grand nombre. Les thèmes utilisés au féminin des adjectifs en -EUR et en -TEUR sont en -euse et en -trice. On prédit donc que leurs dérivés doivent être en -osité (ou -eusité) et en -tricité. C'est ce que confirment les formes trouvées sur la Toile (cf. conductricité et tromposité). Nous voyons là un bon argument en faveur de l'idée que les relations entre formes s'établissent en surface et non par l'intermédiaire d'une forme sous-jacente unique hypothétique. Plus précisément, nous plaidons pour la reconnaissance d'un Thème L(atin) à côté du Thème 1 (celui du masculin) et du Thème 2 (celui du féminin et des dérivés « populaires »).

Certains dérivés de noms en -EUR et en -TEUR sont en -tor et en -or (e.g. modératorat, entraînorat). Au lieu de voir là une objection à l'idée que le Thème $\mathrm{L}$ de l'adjectif est relié à son Thème 2 , nous considérons que celui-ci est distinct du Thème 2 du substantif. Les substantifs en -EUSE et en -TRICE sont des lexèmes à part entière dérivés des substantifs en -EUR et en -TEUR correspondants et non des flexions de ces lexèmes. Dans les substantifs à finale consonantique, le Thème 2 ne se différencie pas du Thème 1. D'où modératorat et entraînorat. Les adverbes en -ment dérivés de noms et d'adjectifs en -EUR et en -TEUR confirment ce point : construits sur le Thème 2 de l'adjectif, les déadjectivaux sont en -eusement et -tricement (cf. trompeusement, motricement), au lieu que les dénominaux sont en -eurement et -teurement quand ils dérivent d'un nom masculin et en -eusement et -tricement quand ils dérivent d'un nom féminin.

La plupart des formes nouvelles utilisées dans cette contribution sont conformes au jugement de l'auteur. Mais des jugements de ce type sont trop volatils pour faire foi. Et, dans certains cas, les jugements individuels ne représentent qu'une des solutions admises par la langue. Comme souvent, il était imprescindible d'accumuler les attestations de formes nouvelles.

\section{Références bibliographiques}

Bonami, O., \& G. Boyé (2003a). Supplétion et classes flexionnelles dans la conjugaison du français. Langages, 152, 102-126.

Bonami, O., \& G. Boyé (2003b). La nature morphologique des allomorphies conditionnées : les formes de liaison des adjectifs en français. In B. Fradin, G. Dal, N. Hathout, F. Kerleroux, M. Plénat \& M. Roché (éds), Les unités morphologiques [= Silexicales 3], SILEX, Université de Lille 3, Lille, 169-178. 
Bonami, O., \& G. Boyé (2005). Construire le paradigme d'un adjectif. Recherches linguistiques de Vincennes, 34, 7798.

Bonami, O., G. Boyé et J. Tseng. (2005). Sur la grammaire des consonnes latentes. Langages, 158, 89-100.

Bonami, O., G. Boyé \& F. Kerleroux (à paraître). L'allomorphie radicale et la relation flexion-construction. In B. Fradin, F. Kerleroux \& M. Plénat (éds), Aperçus de morphologie du français, Saint-Denis : Presses Universitaires de Vincennes.

Boyé, G. (2000). Problèmes de morpho-phonologie verbale en français, en espagnol et en italien. Thèse de doctorat, Université Paris 7.

Boyé, G., \& M. Plénat (à paraître). L'allomorphie radicale dans les lexèmes adjectivaux du français. Le cas des adverbes en -ment. In B. Tranel (ed.), Understanding Allomorphy. Perspectives from Optimality Theory, Londres, Equinox Publishing.

Dell, F., \& E.O. Selkirk (1978). On a Morphologically Governed Vowel Alternation in French. In S.J. Keyser (ed.), Recent Transformational Studies in European Languages, Cambridge, MA : MIT Press, 1-51.

Hathout, N., F. Montermini \& L. Tanguy (2008). Extensive data for morphology: using the World Wide Web. Journal of French Language Studies, 18, 67-85.

Hathout, N., F. Namer, M. Plénat \& L. Tanguy (à paraître). La collecte et l'utilisation des données en morphologie. In B. Fradin, F. Kerleroux \& M. Plénat (éds), Aperçus de morphologie du français. Saint-Denis, Presses Universitaires de Vincennes.

Morin, Y.-C. (1987). Remarques sur l'organisation de la flexion des verbes en français. ITL, Review of Applied Linguistics, 77-78, 13-91.

Morin, Y.-C. (1992). Un cas méconnu de la déclinaison de l'adjectif français : les formes de liaison de l'adjectif antéposé . In André Clas (éd.), Le mot, les mots, les bons mots, 233-250. Montréal : Presses de l'Université de Montréal.

Pichon, E. (1942). Les principes de la suffixation en français. Paris : d'Artrey.

Plénat, M. (ce volume). La liaison « obligatoire » avec et sans enchaînement.

Roché, M. (en préparation). Propositions en morphologie lexicale. À paraître dans les Carnets de grammaire.

Schane, S.A. (1968). French Phonology and Morphology. Cambridge, MA : MIT Press.

\footnotetext{
${ }^{1}$ Néanmoins, comme le dit Pichon (1942: 48), du point de vue de l'amateur de beau langage, « [il] y a toujours de la gaucherie à attacher un suffixe savant à un radical de la souche authentique ».

${ }^{2}$ Je n'ai testé que les adjectifs qui me paraissaient les plus aptes à donner un dérivé en -ité

${ }^{3}$ Deux seulement des 4 occurrences de motorité m'ont semblé dériver vraisemblablement de l'adjectif MOTEUR; les deux autres dérivent probablement du substantif.

${ }^{4}$ Curieusement, ils sont flanqués de bimotricité et de quadrimotricité, dont la présence étonne. En effet, dans les exocentriques bimoteur et quadrimoteur, moteur est un nom et non un adjectif. Il n'y a pas de raison " grammaticale » à ce qu'une finale -moteur qui ne représente pas la «tête » de l'adjectif et qui n'appartient pas elle-même à la catégorie Adjectif soit représentée par motric- dans un dérivé en -ité. On trouve cependant des attestations de bimotrice et de quadrimotrice comme féminins de bimoteur et de quadrimoteur. Il faut donc croire que, pour certains locuteurs, le Thème 2 de ces adjectifs est en tris.

${ }^{5}$ Cette réserve concerne modératricité, dont les deux occurrences me semblent dériver du substantif féminin modératrice.

${ }^{6}$ Je n'ai testé que les adjectifs qui me paraissaient les plus aptes à donner un dérivé en -ité.

${ }^{7}$ Schane (1968) propose une règle de rhotacisation qui s'appliquerait au suffixe -eur $\sim$-euse, mais je connais peu de collègues qui seraient prêts à reprendre cette règle à leur compte. Je ne connais pas non plus de proposition tendant à faire de l'alternance -teur $\sim$-trice le résultat d'une règle phonologique.
} 
ISBN 978-2-7598-0358-3, Paris, 2008, Institut de Linguistique Française

\section{Morphologie}

DOI $10.1051 / \mathrm{cmlf08306}$

${ }^{8}$ Ce sont pour la plupart des emprunts à l'anglais dont la finale peut aussi se prononcer -عr, d'où des dérivés comme leadariat, supportariat, webmestariat.

${ }^{9}$ Michel Roché me signale néanmoins que l'on trouve mérétricat, et puéricultriçat ne me paraît pas totalement exclu. 